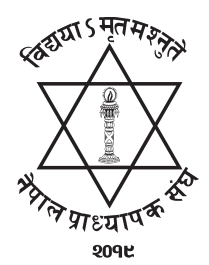

NJ: NUTA

\title{
Urinary Tract Infection Due to Non-fermentative Gram-Negative Bacilli in Tribhuvan University Teaching Hospital, Kathmandu, Nepal
}

\author{
Santosh Kumar Yadav ${ }^{1^{*}}$, Uday Kant Jha ${ }^{2}$ and Jeevan Bahadur Sherchand ${ }^{3}$ \\ ${ }^{1}$ Department of Microbiology, Rajarshi Janak University, Nepal \\ ${ }^{2}$ Department of Hematology, Rajarshi Janak University, Nepal \\ ${ }^{3}$ Department of Microbiology, Institute of Medicine, Tribhuvan University, Nepal \\ "Corresponding authour Email: santooshydv@gmail.com
}

\begin{abstract}
Urinary tract infections (UTIS) are the second most common type of bacterial infection of the body affecting humans throughout their lifetime. They are a frequent cause of nosocomial infection in many hospitals. Therefore, this study was designed to isolate and identify the non-fermentative Gram-negative bacilli (NFGNB) causing UTI in hospitalized patients and determine their antibiotic susceptibility profile. This study was carried in Tribhuvan University Teaching Hospital, Nepal among hospitalized patients. The urine sample was cultured and the antibiotic susceptibility profile of isolated NFGNB was determined by standard microbiological procedures. Among the total of 49 NFGNB isolates, Acinetobacter species ( $n=21,42.9 \%)$ and Pseudomonas aeruginosa $(n=21,42.9 \%)$ were the major isolates and the remaining was Burkholderia cepacia complex ( $n=7,14.3 \%)$. These isolates were found resistant to commonly used antibiotics. From the present study, it is clear that NFGNB are an important bacterial pathogen capable of producing UTI in hospitalized patients.
\end{abstract}

Key words: Acinetobacter, burkholderia cepacia, non-fermentative Gram-negative bacilli, pseudomonas, urinary t ract infection

\section{Introduction}

Urinary tract infections (UTIs) are a serious health problem affecting millions of people each year. Infections of the urinary tract are the second most common type of bacterial infection of the body affecting humans throughout their lifetime (Mittal, Aggarwal, \& Sharma, 2009). They are a frequent cause of morbidity in outpatients as well as a most frequent cause of nosocomial infection in many hospitals. In hospitalized patients, UTIs may represent $25-50 \%$ of infections overall. Escherichia coli is the commonest urinary pathogen accounting for over $80 \%$ of community-acquired infection (Meharwal, Taneja, Sharma, \& Sharma, 2002). Urinary tract infections are responsible for a considerable economic burden for healthcare facilities and national economies; additionally, they also have a substantial economic impact, as they result in lost working days (Gajdács, Burián, \& Terhes, 2019).

Non-fermentative Gram-negative bacilli (NFGNB) or non-fermenters are aerobic Gramnegative bacilli that can survive and grow in an oxygenated environment and are incapable of fermenting 
glucose and utilizing it as a sole source of energy (Berwal et al., 2020). In the hospital environment, non-fermenters may be isolated from instruments such as ventilator machines, humidifiers, mattresses, and other equipment as well as from the skin of healthcare workers (Mellmann et al., 2009). The most common genera belonging to this heterogeneous group of bacteria are Pseudomonas species, Acinetobacter species, Burkholderia species, Alkaligenes species, Stenotrophomonas maltophilia, etc (Bhargava, Kar, \& Saha, 2015). Non-fermenters previously considered as commensals or contaminants are emerging as important nosocomial pathogens because of their frequent isolation from clinical specimens of hospitalized patients and their association with the disease (Arora, Aggarwal, \& Sofat, 2003). The NFGNB vary in their potential to cause infections and antibiotic susceptibility. These organisms have been implicated in causing respiratory tract infections, bacteremia, sepsis, wound infections, UTIs, etc (Gajdács et al., 2019; Meharwal et al., 2002).

There is an increasing appreciation for the pathogenic role of NFGNB in urinary tract infections, particularly in children and in patients, who are present with underlying factors that predispose them to the development of complicated UTIs (Jiménez-Guerra et al., 2018). Non-fermenters are being increasingly reported in significant bacteriuria cases over the years indicating their potential in causing UTIs. It is also a well-known fact that most of these NFGNB are resistant to commonly used antibiotics for treatment (Gupta, Yadav, \& Joshi, 2002). Various studies have been done to know the prevalence of these organisms. Isolation rates for NFGNB in urine samples was $10.8 \%-11.0 \%$ with the distribution of isolates as 72.0\%-87.0\% Pseudomonas aeruginosa, followed by $10.4 \%-18.0 \%$ Acinetobacter baumannii, and $0.77 \%$ Acinetobacter lwoffii (Solanki et al., 2015). Therefore, this study was designed to isolate and identify the NFGNB causing UTI in hospitalized patients and determine their antibiotic susceptibility profile.

Materials and methods

This study was carried in the department of microbiology, Tribhuvan University Teaching Hospital, Kathmandu, Nepal from January 2017 to December 2017 among hospitalized patients suspected with UTI. The ethical approval for the study was obtained from the Institutional Review Board of the Institute of Medicine (IRB-IOM), Tribhuvan University. The urine sample was collected by aseptic technique from the patients and transported to microbiology laboratory and was processed for isolation and identification of NFGNB according to the standard procedures of the American Society for Microbiology (ASM) (Isenberg, 2007).

The urine samples were inoculated on cysteine lactose electrolyte deficient (CLED) agar plate by semi-quantitative continuous culture method. The inoculated agar plates were incubated at $37^{\circ} \mathrm{C}$ for 24 hours in an aerobic atmosphere. The significant bacterial growth was indicated by a bacterial growth of $\geq 10^{5} \mathrm{CFU} / \mathrm{ml}$. Identification of significant bacterial isolates was done by standard microbiological techniques that involved Gram's staining, motility test, catalase test, oxidase test, triple sugar iron agar test, citrate utilization test, urea hydrolysis test, gelatin liquefaction test, arginine dihydrolase test, lysine decarboxylase test, pigment production, and other biochemical properties (Isenberg, 2007).

The antibiotic sensitivity test (AST) of the significant NFGNB isolates was performed by the standard disk diffusion technique of the Kirby-Bauer method as recommended by the Clinical and Laboratory Standard Institute (CLSI). For this, 3-5 well-isolated colonies of bacteria were transferred into $5 \mathrm{ml}$ of peptone water and incubated at $37^{\circ} \mathrm{C}$ until its turbidity matched with $0.5 \mathrm{McFarland}$ 
standard. A sterile cotton swab was dipped into the suspension and the swab was rotated on the inner wall of the tube to remove excess inoculum from the swab. Then the entire surface of a Mueller Hinton Agar (MHA) plate was inoculated with the swab to produce a lawn culture. The plates were dried for 10 minutes at room temperature and then antibiotic disks were placed on the surface of the agar plate and incubated in ambient air for $16-18$ hours at $37^{\circ} \mathrm{C}$. The NFGNB isolates were tested against recommended concentrations of piperacillin, piperacillin-tazobactam, ceftazidime, cefepime, meropenem, gentamicin, amikacin, ciprofloxacin, levofloxacin, cotrimoxazole, doxycycline, and polymyxin B (Himedia, India) as per CLSI guidelines. The results were compared with a zone size interpretative chart to determine the bacterial susceptibility towards different antimicrobial agents in terms of sensitive, resistant, and intermediate sensitive (CLSI, 2016).

The data generated during the study were analyzed using SPSS 16.0 version and interpreted according to frequency distribution and percentage.

\section{Results}

During the study period, a total 2614 urine sample was collected from the hospitalized patients suspected with UTI. Among the total samples, 280 showed significant bacterial growth $\left(\geq 10^{5} \mathrm{CFU} / \mathrm{ml}\right)$ which included 49 (17.5\%) NFGNB isolates.

Among 49 NFGNB isolates, 27 (55.1\%) were isolated from female patients while $22(44.9 \%)$ were isolated from male patients with a female to male ratio of 1.23 . The age-wise distribution of NFGNB isolates causing UTI in hospitalized patients is shown in Table 1.

Among total NFGNB isolates, the majority were isolated from patients of the surgical ward while the least number isolated from the pediatrics ward (Table 2).

The total number of NFGNB isolates comprised of three genera which included Acinetobacter species ( $\mathrm{n}=21,42.9 \%)$, Pseudomonas aeruginosa $(\mathrm{n}=21,42.9 \%)$ and the least number was Burkholderia cepacia complex $(\mathrm{n}=7,14.3 \%)$.

The antibiotic susceptibility profile of NFGNB isolates causing UTI in hospitalized patients is shown in Table 3.

Table 1. Age-wise distribution of NFGNB isolates causing UTI in hospitalized patients

\begin{tabular}{cccc}
\hline $\begin{array}{c}\text { Age group } \\
\text { (years) }\end{array}$ & Female & Male & Total \\
\cline { 2 - 4 } $\mathbf{5 1 5}$ & $2(4.1)$ & $1(2.0)$ & $3(6.1)$ \\
$\mathbf{1 6 - 3 2}$ & $15(30.6)$ & $8(16.3)$ & $23(46.9)$ \\
$\mathbf{3 3 - 4 8}$ & $7(14.3)$ & $5(10.2)$ & $12(24.5)$ \\
$\mathbf{4 9 - 6 4}$ & $2(4.1)$ & $3(6.1)$ & $5(10.2)$ \\
$\mathbf{2 6 5}$ & $1(2.0)$ & $5(10.2)$ & $6(12.2)$ \\
\hline Total & $27(55.1)$ & $22(44.9)$ & $49(100)$ \\
\hline
\end{tabular}


Table 2. Ward wise distribution of NFGNB isolates causing UTI in hospitalized patients

\begin{tabular}{lcc}
\hline Wards & Number of NFGNB isolates & Percentage \\
\hline Intensive care unit & 6 & 12.2 \\
Medical & 9 & 18.4 \\
Surgical & 16 & 32.7 \\
Pediatrics & 2 & 4.1 \\
Maternity & 13 & 26.5 \\
Orthopedics & 3 & 6.1 \\
\hline Total & 49 & 100.0 \\
\hline
\end{tabular}

Table 3. Antibiotic susceptibility profile of NFGNB isolates causing UTI

\begin{tabular}{|c|c|c|c|c|c|c|}
\hline \multirow[t]{2}{*}{ Antibiotics } & \multicolumn{2}{|c|}{$\begin{array}{c}\text { Acinetobacter species } \\
(\mathrm{n}=21)\end{array}$} & \multicolumn{2}{|c|}{$\begin{array}{c}\text { P. aeruginosa } \\
(\mathrm{n}=\mathbf{2 1})\end{array}$} & \multicolumn{2}{|c|}{$\begin{array}{c}\text { B. cepacia complex } \\
(n=7)\end{array}$} \\
\hline & S (\%) & R (\%) & S (\%) & R (\%) & S (\%) & R (\%) \\
\hline Piperacillin & $7(33.3)$ & $14(66.7)$ & $6(28.6)$ & $15(71.4)$ & - & - \\
\hline $\begin{array}{l}\text { Piperacillin- } \\
\text { tazobactam }\end{array}$ & $9(42.9)$ & $12(57.1)$ & $9(42.9)$ & $12(57.1)$ & - & - \\
\hline Ceftazidime & $8(38.1)$ & $13(61.9)$ & $3(14.3)$ & $18(85.7)$ & $0(0)$ & $7(100)$ \\
\hline Cefepime & $7(33.3)$ & $14(66.7)$ & $2(9.5)$ & $19(90.5)$ & - & - \\
\hline Meropenem & $10(47.6)$ & $11(52.4)$ & $8(38.1)$ & 13 (61.9) & $4(57.1)$ & $3(42.1)$ \\
\hline Gentamicin & $10(47.6)$ & $11(52.4)$ & $8(38.1)$ & $13(61.9)$ & - & - \\
\hline Amikacin & $11(52.4)$ & $10(47.6)$ & $14(66.7)$ & $7(33.3)$ & - & - \\
\hline Ciprofloxacin & $8(38.1)$ & 13 (61.9) & $2(9.5)$ & $19(90.5)$ & - & - \\
\hline Levofloxacin & $9(42.9)$ & $12(57.1)$ & $4(19.0)$ & $17(81.0)$ & $1(14.3)$ & $6(85.7)$ \\
\hline Cotrimoxazole & $5(23.8)$ & $16(76.2)$ & - & - & $7(100)$ & $0(0)$ \\
\hline Doxycycline & $14(66.7)$ & $7(33.3)$ & - & - & $3(42.9)$ & $4(57.1)$ \\
\hline Polymyxin B & $21(100)$ & $0(0)$ & $21(100)$ & $0(0)$ & - & - \\
\hline
\end{tabular}




\section{Discussion}

The importance of isolation and identification of NFGNB has been increased in the last decade after more and more reports are correlating them with either infection outbreaks in hospitals or healthcare-associated infections. In the current study, we have searched for various non-fermenters causing UTIs in patients admitted to different wards of Tribhuvan University Teaching Hospital, Kathmandu, Nepal. A total of 49 NFGNB were isolated representing 17.5\% of significant culturepositive samples and $1.9 \%$ of total urine samples. Nautiyal, Jauhari, Goel, \& Mahawal (2014) also reported 17.4\% NFGNB among culture-positive urine samples which are similar to our result. Berwal et al., (2020) reported 0.6\% NFGNB out of total urine samples and 33.9\% NFGNB among culturepositive samples while Sharma, Vyas, Sinha, \& Mathur (2014) documented 7.8\% NFGNB out of total urine samples and $13.8 \%$ NFGNB among culture-positive samples. These variations in the isolation rate of NFGNB in different hospitals might be because of the infection control practices of respective hospitals.

The demographic data in our study showed that the age group most commonly affected by NFGNB infections was between 16-32 years which is in accordance with the study done by Malini, Deepa, Gokul, \& Prasad (2009) but a study from Iran showed a higher rate of NFGNB infections in patients having more than 60 years age because higher age group patients have a lower defense against these bacteria and NFGNB get a chance to infect them (Rahbar, Mehragan, \& Akbari, 2010). The pattern of UTI due to NFGNB in the patients of different wards was evaluated in this study. Higher numbers of NFGNB were isolated from patients of the surgical ward followed by maternity ward and medical ward. But another study has reported a higher incidence of NFGNB in intensive care unit (ICU) patients (Eltahawy \& Khalaf, 2001). It has been stated that nosocomial infection rates in ICUs are many times higher than elsewhere in hospitals as ICUs are the hub of severely ill patients, who are most prone to opportunistic infections by NFGNB.

The isolated NFGNB were identified phenotypically in this study. Acinetobacter species and Pseudomonas aeruginosa represent major isolates (each 42.9\%) and the remaining isolate was Burkholderia cepacia complex (14.3\%). Acinetobacter species as a predominant NFGNB in hospitalized patients was also concluded by Parajuli et al., (2017) and Sah et al., (2014) in the same hospital while Malini et al., (2009) and Sharma et al., (2014) recognized Pseudomonas aeruginosa as predominant NFGNB isolate.

Antimicrobial resistance is a global problem in health care facilities (WHO, 2014). In this study, many NFGNB isolates causing UTI in hospitalized patients were found resistant to different antimicrobial agents. The antibiotic susceptibility test in our study confirmed an alarming percentage of resistance exhibited by NFGNB. Acinetobacter species were found significantly resistant to betalactams, fluoroquinolones, aminoglycosides, and cotrimoxazole. More than $60 \%$ of Acinetobacter species were found resistant to piperacillin, ceftazidime, cefepime, ciprofloxacin, and cotrimoxazole. Mahajan \& Mahajan (2016) also reported 88\% Acinetobacter species resistant to ceftazidime, $87 \%$ to cefepime, $78 \%$ to ciprofloxacin, and $75 \%$ to cotrimoxazole. The increasing emergence of aminoglycoside-resistant strains is also a cause of major concern. Our result on gentamicin resistance was $52.4 \%$ which is similar to the previous study from the same hospital (Mishra, Rijal, \& Pokhrel, 2013). However, none of the Acinetobacter species were found resistant to Polymyxin B which is 
similar to the result of Shrestha et al., (2015). Among the antibiotics tested, 85.7\% of Pseudomonas aeruginosa isolates were resistant to ceftazidime, $61.9 \%$ to gentamicin, $71.4 \%$ to piperacillin, and $90.5 \%$ to ciprofloxacin. All isolates were susceptible to polymyxin B. A similar resistance rate was reported by Parajuli et al., (2017) from the same hospital, Fatima, Naqvi, Khaliq, Perveen, \& Jabeen (2012) from Pakistan, and Xie et al., (2011) from China. Antimicrobial resistance was also found common in isolates of Burkholderia cepacia complex. All the isolates were found susceptible to cotrimoxazole, $42.9 \%$ to doxycycline and only $14.3 \%$ were sensitive to levofloxacin. A similar pattern of susceptibility was reported from the study of Parajuli et al., (2017).

In this study, polymyxin B showed excellent effect against Acinetobacter species and Pseudomonas aeruginosa isolates, and cotrimoxazole was found to be effective against Burkholderia cepacia complex. However, the present study showed a high antibiotic resistance rate against commonly used antibiotics and is a disadvantage for the healthcare system in countries like Nepal as it can greatly affect the management of hospitalized patients.

\section{Conclusion}

The present study concludes that the NFGNB, though considered as commensals or contaminants previously, are important bacteria capable of producing UTI in hospitalized patients. The variability in the antibiotic sensitivity profile of NFGNB highlights the need for their identification and to monitor their susceptibility profile as it will help in the proper management of UTI caused by them. More importantly, these bacteria have a great capacity to survive in the healthcare environment so improved infection control measures and antibiotic stewardship programs will be needed to prevent or decrease the emergence and spread of antibiotic-resistant NFGNB in hospitals.

\section{References}

Arora, U., Aggarwal, A., \& Sofat, S. (2003). Non-fermenters in human infections. Indian J Pathol Microbiol, 46(2), 265-267. Retrieved from

Berwal, A., Shobha, K., Gupta, R., Gupta, K., Ashraf, A., Kumar, A., ... Sonali. (2020). Non-fermenting gram negative bacteria as uropathogens in causing urinary tract infection and its antimicrobial susceptibility pattern at a Tertiary Care Centre of South India. Journal of Pure and Applied Microbiology, 14(3), 2033-2038.

Bhargava, D., Kar, S., \& Saha, M. (2015). Prevalence of Non-Fermentative Gram Negative Bacilli Infection in Tertiary Care Hospital in Birgunj, Nepal. International Journal of Current Microbiology and Applied Sciences, 4(7), 301-307.

CLSI. (2016). Performance Standards for Antimicrobial Susceptibility Testing; Twenty-Sixth Informational Supplement. CLSI Document M100S (26th ed.). Clinical and Laboratory Standards Institute, Wayne, PA, USA.

Eltahawy, A., \& Khalaf, R. (2001). Antibiotic resistance among Gram-negative non-fermentative bacteria at a teaching hospital in Saudi Arabia. Journal of Chemotherapy, 13(3), 260-264.

Fatima, A., Naqvi, S., Khaliq, S., Perveen, S., \& Jabeen, S. (2012). Antimicrobial susceptibility pattern of clinical isolates of Pseudomonas aeruginosa isolated from patients of lower respiratory tract infections. Springer Plus, 1(1), 70.

Gajdács, M., Burián, K., \& Terhes, G. (2019). Resistance levels and epidemiology of non-fermenting gram-negative bacteria in urinary tract infections of inpatients and outpatients (RENFUTI): A

NUTA JOURNAL, 7 (1 \& 2), 2020 : ISSN: 2616 - 017x 
10-year epidemiological snapshot. Antibiotics, 8(3), 1-13.

Gupta, V., Yadav, A., \& Joshi, R. (2002). Antibiotic resistance pattern in uropathogens. Indian Journal of Medical Microbiology, 20, 96-98.

Isenberg, H. (2007). Clinical Microbiology Procedure Handbook (Second Edi). American Society for Microbiology (ASM), Washington, DC, USA.

Jiménez-Guerra, G., Heras-Cañas, V., Gutiérrez-Soto, M., Aznarte-Padial, M., Expósito-Ruiz, M., Navarro-Marí, J., \& Gutiérrez-Fernández, J. (2018). Urinary tract infection by acinetobacter baumannii and pseudomonas aeruginosa: Evolution of antimicrobial resistance and therapeutic alternatives. Journal of Medical Microbiology, 67(6), 790-797.

Mahajan, R., \& Mahajan, B. (2016). Isolation and Identification of Non Fermenting Gram Negative Bacilli in A Tertiary Care Hospital. Scholars Journal of Applied Medical Sciences, 4(3D), 872-876.

Malini, A., Deepa, E., Gokul, B., \& Prasad, S. (2009). Nonfermenting gram-negative bacilli infections in a tertiary care hospital in Kolar, Karnataka. Journal of Laboratory Physicians, 1(2), 62-66.

Meharwal, S., Taneja, N., Sharma, S., \& Sharma, M. (2002). Complicated nosocomial UTI caused by nonfermenters. Indian Journal of Urology, 18(2), 123-128.

Mellmann, A., Bimet, F., Bizet, C., Borovskaya, A., Drake, R., Eigner, U., ... Harmsen, D. (2009). High Interlaboratory Reproducibility of Matrix-Assisted Laser Desorption Ionization-Time of Flight Mass Spectrometry-Based Species Identification of Nonfermenting Bacteria. Journal of Clinical Microbiology, 47(11), 3732-3734.

Mishra, S., Rijal, B., \& Pokhrel, B. (2013). Emerging threat of multidrug resistant bugs-Acinetobacter calcoaceticus baumannii complex and Methicillin resistant Staphylococcus aureus. BMC Research Notes, 6(98), 1-6.

Mittal, R., Aggarwal, S., \& Sharma, S. (2009). Urinary tract infections caused by Pseudomonas aeruginosa : A minireview. Journal of Infection and Public Health, 2, 101-111.

Nautiyal, S., Jauhari, S., Goel, N., \& Mahawal, B. (2014). Current Trend of Nonfermenting Gram Negative Bacilli in a Tertiary Care Hospital in Dehradun, Uttarakhand. International Journal of Advanced Research, 2(2), 322-328.

Parajuli, N. P., Acharya, S. P., Mishra, S. K., Parajuli, K., Rijal, B. P., \& Pokhrel, B. M. (2017). High burden of antimicrobial resistance among gram negative bacteria causing healthcare associated infections in a critical care unit of Nepal. Antimicrobial Resistance and Infection Control, 6(1), 67.

Rahbar, M., Mehragan, H., \& Akbari, N. (2010). Prevalence of Drug Resistance in Nonfermenter Gram-Negative Bacilli. Iranian Journal of Pathology, 5(2), 90-96.

Sah, M., Mishra, S., Ohora, H., Kirikae, T., Sherchand, J., Rijal, B., \& Pokhrel, B. (2014). Nosocomial Bacterial Infection and Antimicrobial Resistant Pattern in a Tertiary Care Hospital in Nepal. Journal of Institute of Medicine, 36(3).

Sharma, D., Vyas, N., Sinha, P., \& Mathur, A. (2014). Non fermentative gram negative bacilli as nosocomial pathogens: Identification and antibiotic sensitivity in clinical samples of indoor patients. Nepal Journal of Medical Sciences, 03(02), 101-105. 
Shrestha, Tada, T., Shrestha, B., Kirikae, T., Rijal, B., Pokhrel, B., \& Sherchand, J. (2015). Phenotypic characterization of multidrug-resistant Acinetobacter baumannii with special reference to metallo- $\beta$-lactamase production from the hospitalized patients in a tertiary care hospital in Nepal. Journal of Institute of Medicine, 37(3), 3-10.

Solanki, R., Dawar, R., Aggarwal, D. K., Rani, H., Imdadi, F., \& Jasuja, S. (2015). Nonfermenting Gram-Negative Bacilli and Urinary Tract Infection -Sorting the Mystery of Infections Caused. Medical Microbiology and Diagnosis, 4(4), 1-3.

WHO. (2014). Antimicrobial resistance: global report on surveillance 2014. In World Health Organization.

Xie, D., Xiong, W., Lai, R., Liu, L., Gan, X., Wang, X., ... Nie, S. (2011). Ventilator-associated pneumonia in intensive care units in Hubei Province, China: a multicentre prospective cohort survey. The Journal of Hospital Infection, 78(4), 284-188. 